\title{
Using High Resolution X-ray Computed Tomography to Create an Image Based Model of a Lymph Node
}

\author{
L. J. Cooper ${ }^{\mathrm{a}, *}$, B. Zeller-Plumhoff ${ }^{\mathrm{a}}$, G. F. Clough $^{\mathrm{b}}$, B. Ganapathisubramania ${ }^{\mathrm{a}}$, \\ T. Roose ${ }^{\mathrm{a}}$ \\ ${ }^{a}$ Faculty of Engineering and the Environment, University of Southampton, Highfield \\ Campus, Southampton, SO17 1BJ, UK \\ ${ }^{b}$ Faculty of Medicine, University of Southampton, Southampton General Hospital, \\ Southampton, SO16 6YD, UK
}

\begin{abstract}
Lymph nodes are an important part of the immune system. They filter the lymphatic fluid as it is transported from the tissues before being returned to the blood stream. The fluid flow through the nodes influences the behaviour of the immune cells that gather within the nodes and the structure of the node itself. Measuring the fluid flow in lymph nodes experimentally is challenging due to their small size and fragility. In this paper, we present high resolution $\mathrm{X}$-ray computed tomography images of a murine lymph node. The impact of the resulting visualized structures on fluid transport are investigated using an image based model. The high contrast between different structures within the lymph node provided by phase contrast X-ray computed tomography reconstruction results in images that, when related to the permeability of the lymph node tissue, suggest an increased fluid velocity through the interstitial channels in the lymph node tissue. Fluid taking a direct path from the afferent to the efferent lymphatic vessel, through the centre of the node, moved faster than the fluid that flowed around the periphery of the lymph node. This is a possible mechanism for particles being moved into the cortex.
\end{abstract}

Keywords: Lymphatic System, Image based modelling, Porous media

\footnotetext{
* Corresponding author

Email address: laura.cooper@soton.ac.uk (T. Roose)
}

Preprint submitted to Journal of ${ }^{A} T_{E} X$ Templates

March 21, 2018 


\section{Introduction}

Lymph nodes are a site for immune cell transfer between blood and lymphatic fluid, where antigen sensing and immune cell activation takes place. Lymph nodes have been imaged using histology for many years [1, 2, 3, 4].

5 However, histology can only ever give 2D structural information about this $3 \mathrm{D}$ organ. Some of the structures identified within the lymph nodes are:

- a fibrous outer capsule encasing the whole node

- a low resistance pathway for fluid just under the capsule, known as the subcapsular sinus;

- an area of dense lymphoid tissue, called the cortex;

- and the when the fluid leaves the cortex and subcapsular sinus, if it is not reabsorbed via the blood vessels, it enters the medullary sinuses before exiting the node through an efferent lymphatic vessel.

The structure of lymph nodes can be used to theorise on the behaviour of fluid flow through the nodes [5. The fluid flow in lymph nodes has been shown to affect the arrangement of the reticular cell network [6] and to influence the migration of immune cells $[7,8$. The fluid flow also determines the concentration of proteins in the lymphatic fluid [9, 10, 11]. Understanding the pathways of fluid flow through the nodes will contribute to our knowledge of immune cell migration, development of lymph node structures, protein transfer and cancer metastasis [12. Experimental investigations of fluid flow through lymph nodes is challenging due to the lymph node's small size and fragility. In this paper, we combine high resolution imaging with computational modelling to demonstrate how the internal structures within the node can influence the fluid flow pathways.

Mayer et al. [13] used selective plane illumination microscopy (SPIM) to visualise the high endothelial venules and dendritic cells in two lymph nodes in 3D. The SPIM images of these lymph nodes have also been used to create a 
finite element model of fluid flow through lymph nodes [14]. The model based on the SPIM images was used to estimate properties such as the permeability of the node by fitting to experimental data and showed that the main pathway for fluid flow in the model was through the centre of the node. A limitation of using SPIM images is that fluorescent staining is required to enhance the image contrast of the structures within the node. One consequence of this is that the relation between the permeability of the lymph node tissue and the greyscale of the image may be biased depending on the diffusion of the stain into the tissue. For example, there could be more stain around the outer edges of the node than in the centre.

Other three-dimensional, non-destructive imaging methods exist that do not 40 require soft tissue to be stained to generate image contrast, and thus overcome the limitation of using SPIM images. Namely, high resolution X-ray computed tomography (CT) can be used to visualise the variable density of the lymph node internal tissue structure in 3D. The capability of synchrotron radiation CT has previously been used to generate 3D data sets of soft tissue for subsequent finite element modelling. This is demonstrated by Zeller-Plumhoff et al. [15, 16] in the context of oxygen diffusion in murine muscle. High resolution CT can also enable the analysis of morphological measures in biological systems and their functional importance [16].

In this paper, we use X-ray CT images with the finite element method to create an image based model of fluid flow through a lymph node. We are utilising the relationship between image greyscale and tissue permeability to assess the importance of the structures observed using X-ray CT on lymphatic fluid flow. Two different methods of enhancing image contrast for soft tissue features are used in order to establish which method best enables visualisation of the features of interest. These methods are edge enhancement by direct image reconstruction using the fast reconstruction algorithm Gridrec [17, 18, and phase retrieval with the Paganin single-distance non-iterative phase retrieval algorithm [19] and subsequent reconstruction with Gridrec. The outcome from the phase retrieval images produces results that are consistent with previous 
studies on the internal structure of the node [5]. The methodology presented in this paper can be generalised and used to assess differences between lymph nodes due to, for example, different stages of a disease.

\section{Methods}

\subsection{Sample Preparation, Imaging and Reconstruction}

All animal procedures were in accordance with the regulations of the United Kingdom Animals (Scientific Procedures) Act 1986 and were conducted under Home Office Licence number 70-6457. The study received institutional approval from the University of Southampton Biomedical Research Facility Research Ethics Committee. The mouse was maintained under controlled conditions and at 15 weeks of age, the mouse was sacrificed by cervical dislocation. A mesenteric lymph node $\left(\approx 1 \times 1 \times 2 \mathrm{~mm}^{3}\right)$ was dissected from a male C57BL/6 mouse, fixed in formalin, dehydrated in methylated spirit and embedded in paraffin wax. The lymph node was scanned at the beamline for Tomographic Microscopy and Coherent radiology experiments (TOMCAT) at the Swiss Light

75 Source, Villigen, Switzerland. The imaging was carried out using an energy of $14 \mathrm{keV}$, using a propagation distance between sample and detector of $60 \mathrm{~mm}$. The exposure time was $180 \mathrm{~ms}$ and 1601 projections were taken over $180^{\circ}$ (with additional 32 dark field and 160 flat field images). The resulting voxel size was $(0.65 \mu \mathrm{m})^{3}$. The images were reconstructed using two methods, both in-house so implementations at TOMCAT: the Gridrec algorithm [17, 18] and the Paganin phase retrieval algorithm 19 with Gridrec. A comparison of the two resulting reconstructions can be seen in figure 1. The edge enhancement in the direct Gridrec reconstruction shows small length scale features within the node, which could possibly be the lymphocytes that make up the tissue of the node. The

85 higher contrast of the Paganin reconstruction shows features at the larger length scale. It is possible to distinguish structures that appear to be B cell follicles, lymphatic channels, the dense cortex and less dense paracortex, similar to those seen with histology, e.g. 3. It should be noted that although structures can be 

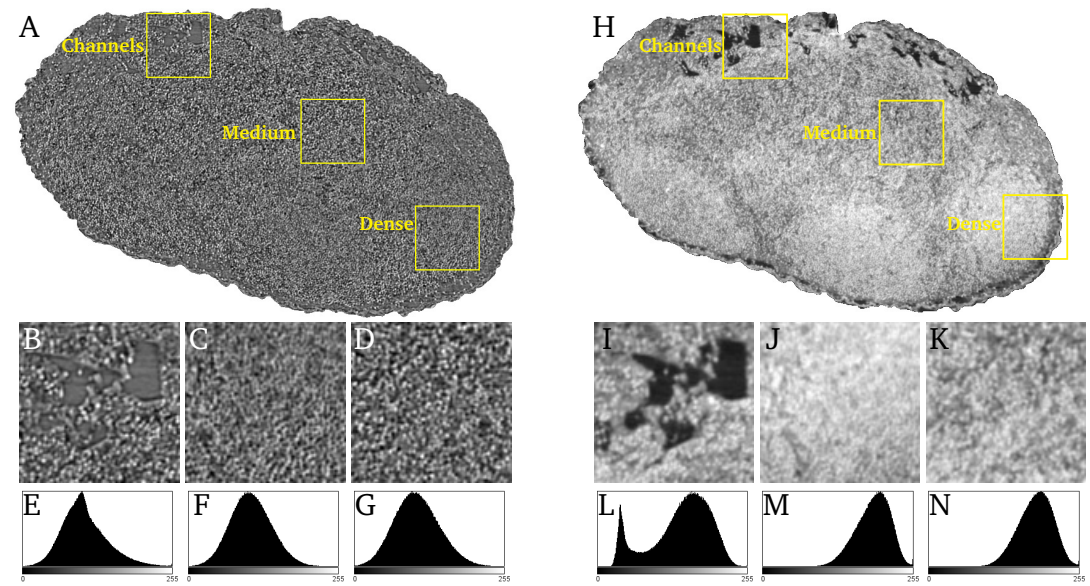

Figure 1: Different regions used to evaluate the effect of areas of the node on the flow pathways in the model for A) Gridrec reconstruction and H) Paganin reconstruction. B and I) Channels from Gridrec and Paganin, respectively; C and J) Dense from Gridrec and Paganin, respectively; D and K), Medium from Gridrec and Paganin, respectively; E and L), Histogram of channels for Gridrec and Paganin, respectively; F and M), Histogram of dense for Gridrec and Paganin, respectively; G and N), Histogram of medium for Gridrec and Paganin, respectively. The squares are all $0.13 \mathrm{~mm}^{2}$.

distinguished due to the contrast of the X-ray CT images, it would be necessary to correlate these images with histology to confirm the identity of these structures. Histology was not undertaken as correlating the 2D histological sections to the 3D image stacks would have required an extra layer of techniques to be applied, trialled and calibrated, that are outside the scope of this paper.

\subsection{Blood Vessel Segmentation and Construction}

Only a proportion of blood vessels appeared visible in the X-ray CT reconstructions. Therefore a vascular tree was constructed computationally. The blood vessels that could be visualised were segmented manually using the magic wand tool on individual slices spaced 5-10 $\mu \mathrm{m}$ apart in Avizo Fire 9.0 (FEI, Hillsboro, OR, USA). Interpolation between the manually segmented slices was used to create continuous blood vessels. The blood vessels were constructed 
computationally. The vessels that could be segmented out were used as inputs to the fractal L-system algorithm used to create the vascular network. Details of the L-system used can be found in Appendix A

The tree was generated using a script in MATLAB and converted into an image stack using macros in Fiji image analysis software [20. A new tree was initiated at the end points of each segmented branch from the images. The 3D line tool from the 3D tool plugin was used to draw white lines of a fixed radius on a stack of black images. The macro could then be run in Fiji to create an image stack of the computationally created blood vessels that was then combined with the masks for the surfaces representing the lymphatic vessel connections and the mask for the outline of the lymph node. Three separate tree realisations were created consisting of 4,5 and 6 levels of branches, respectively, with heading vectors based on the segmented images. Another three trees were created by defining start points and heading vectors randomly. Two of these trees were created with 14 and 28 start points, respectively, and 6 branching levels. All vessels created in these trees had a diameter of $5.2 \mu \mathrm{m}$. The sixth tree was the 28 start point tree where the diameter of the vessels were increased to 10.4 $\mu \mathrm{m}$. The value of $L_{p}$ for each model was calculated from the published value of $L_{p} \times S$ for the WT node from [14], where $S$ is the blood vessel surface area, by dividing by the surface area for the created blood vessel trees. The models with different branching structures were modelled with a constant permeability for the lymph node interstitial space. The value of $L_{p}$ for each tree can be seen in figure 2 .

\subsection{Image Processing} node was segmented by applying a threshold to the phase contrast image stack. The threshold was chosen by manually varying the minimum and maximum values of the histogram so that the whole node was segmented and the background colour was set to black. Any additional material, mainly fatty tissue around the node, was removed from the image manually. The voxel size was 


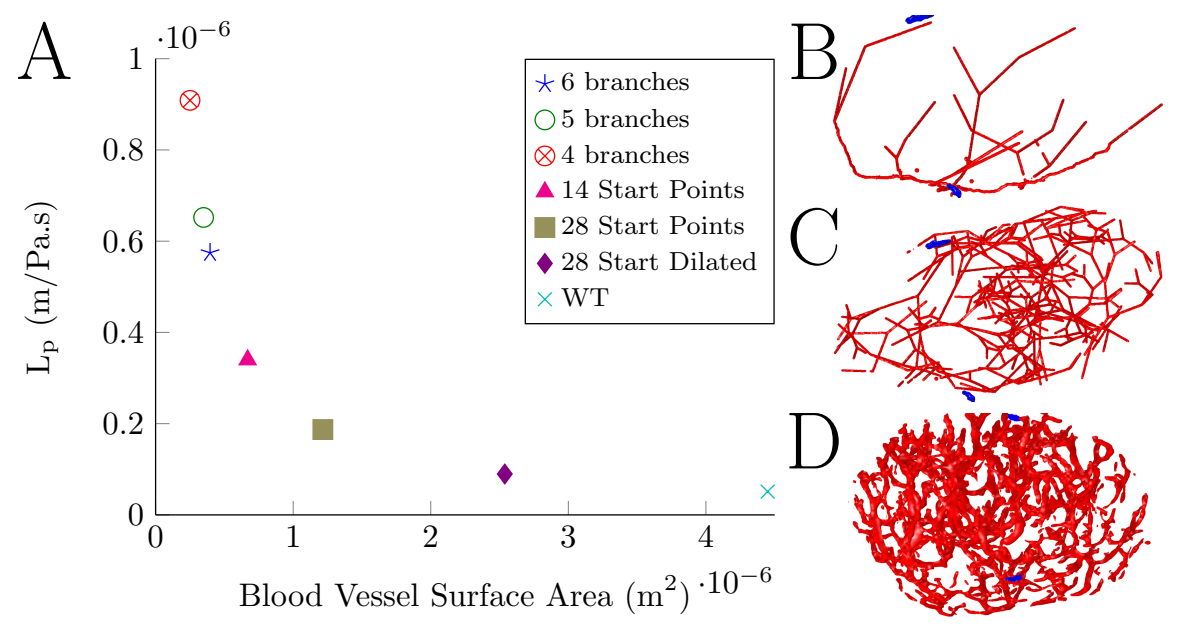

Figure 2: A) Relationship of blood vessel surface area to the value of $L_{p}$, where the value of $L_{p} S=2.27 \times 10^{-13}$ has been assumed and is represented by WT based on the wild type node from Cooper et al. 14. Figures $\mathrm{B}$ and $\mathrm{C}$ show examples of the blood vessel networks used corresponding to 4 branches and 28 Start Points, respectively. The blood vessels are shown in red and the positions of the afferent and efferent lymphatics in blue, with the afferent vessel at the top of the image and the efferent vessel at the bottom. Figure D shows the high endothelial venules for the wild type (WT) node from Cooper et al. 14 for comparison.

binned to $(1.3 \mu \mathrm{m})^{3}$ to decrease the size of the image stack to an eighth of the original to reduce the computational resources required. A mask was created of the segmented node, i.e. a binary image that showed the lymph node geometry without the internal detail. The mask was used to remove everything outside of the node from the Gridrec image stack, so that the node was on a white background, as shown in figure $1 \mathrm{~A}$ and figure $1 \mathrm{H}$. Each slice from Gridrec and phase contrast image stacks were saved as .png files for use in COMSOL 4.4 (COMSOL AB, Sweden). The mask of the computationally created blood vessels was subtracted from the mask of the segmented node, creating binary images that showed the blood vessels as holes within the mask of the node geometry. To segment the afferent and efferent lymphatic vessels the magic wand tool was used in Avizo Fire on the segmented phase stack. This was saved as another mask. The masks of the segmented node with blood vessels and the mask of 
the afferent and efferent lymphatic vessels were imported into ScanIP 4.0 (Simpleware Ltd, UK) for meshing. The whole node was meshed with minimum element size $2.25 \mu \mathrm{m}$ and maximum element size $9 \mu \mathrm{m}$. The mesh produced had the geometry of the lymph node with holes that represented the location of blood vessels. A more detailed description of how to use ScanIP to mesh the lymph node can be found in [14].

\subsection{Modelling of Fluid Flow Through the Lymph Node}

The modelling was carried out following the approach first introduced in Cooper et al. [14. Briefly, the fluid flow through the lymph node interstitium, $\Omega$, is modelled using Darcy's law in combination with the fluid conservation equation,

$$
\mathbf{u}=-\frac{\kappa}{\mu} \nabla p, \quad \mathbf{x} \in \Omega^{C}, \Omega^{L}
$$

$$
\nabla \cdot \mathbf{u}=0, \quad \mathbf{x} \in \Omega^{C}, \Omega^{L}
$$

where $\kappa$ is the interstitial permeability defined using the image stacks,

$$
\begin{aligned}
\kappa= & \left(-k_{0} i m_{1}(x, y)+k_{1}\right) \times(z<1.3 \mu \mathrm{m}) \\
& +\left(-k_{0} i m_{2}(x, y)+k_{1}\right) \times(z \geq 1.3 \mu \mathrm{m}) \times(z<2.6 \mu \mathrm{m}) \\
& +\cdots+\left(-k_{0} i m_{n}(x, y)+k_{1}\right) \times(z \geq 1.3(n-1) \mu \mathrm{m}), \quad \mathbf{x} \in \Omega^{C}, \Omega^{L}
\end{aligned}
$$

where $k_{0}$ and $k_{1}$ relate the grey scales of the images and the permeability linearly. $i m_{n}$ is the nth slice of the image stack, $x, y$ and $z$ and the coordinates of the point in the image stack. Here, $k_{0}=6.2086 \times 10^{-11} \mathrm{~m}^{2}$ and $k_{1}=6.2096 \times 10^{-11} \mathrm{~m}^{2}$, the values are for the wild type murine lymph node after Cooper et al. [14] unless

otherwise stated. $\mathbf{u}$ is the fluid velocity, $\mu=0.0015 \mathrm{~Pa} \cdot \mathrm{s}$ is the dynamic viscosity of the lymphatic fluid [21] and $p$ is the fluid pressure in the node interstitium.

The boundary condition on the blood vessel surfaces was defined using Starling's principle,

$$
\hat{\mathbf{n}}_{b} \cdot \rho \boldsymbol{u}=L_{p}\left[\left(p_{v}-p\right)-\sigma(\Delta \pi)\right], \mathbf{x} \in \Gamma_{b}^{L}
$$


where $\hat{\mathbf{n}}_{b}$ is the vector normal pointing from the surface of the blood vessels toward the interstitum, $\rho=1000 \mathrm{~kg} / \mathrm{m}^{3}$ is the density of the fluid, $L_{p}(\mathrm{~m} / \mathrm{Pa} \cdot \mathrm{s})$ is the hydraulic conductivity of the blood vessel walls and had different values depending on the surface area of the blood vessel tree, $p_{v}=973 \mathrm{~Pa}$ is the pressure inside the blood vessels [11], $\sigma=0.9$ (no units) is the osmotic reflection coefficient [22], and $\Delta \pi=\pi_{v}-\pi_{n}=341 \mathrm{~Pa}$, where $\pi_{v}(\mathrm{~Pa})$ is the plasma colloid osmotic pressure in the blood vessels and $\pi_{n}(\mathrm{~Pa})$ is the node colloid osmotic pressure. The value of $\Delta \pi=341 \mathrm{~Pa}$ was found by fitting the wild type model in Cooper et al. 14 to experimental data. This value is smaller than was approximated by Adair and Guyton [11], $\Delta \pi \approx 2080 \mathrm{~Pa}$ for dog lymph nodes, and the value measured in the skin of mice $\Delta \pi \approx 1500 \mathrm{~Pa}[23$. However, it was found that a higher value of $\Delta \pi$ did not give efferent flow rates that fit well with the experimental data [14]. $\Gamma_{b}^{L}$ is the blood vessel boundary.

In this paper we present two studies; firstly, a mesh density study to optimise the accuracy of the model result while reducing the computational resources required. For this study a cubic subsection of the lymph node is used to reduce the complexity of the model until the mesh parameters have been established. Secondly, we present a study where the whole lymph node is modelled to investigate how the internal structure of the node affects the pathways of fluid through the node. One side of the cube, $\Lambda_{1}$, has the boundary condition,

$$
\hat{\mathbf{n}}_{1} \cdot \rho \mathbf{u}=\frac{F_{1}}{A_{1}}, \mathbf{x} \in \Gamma_{1}^{C},
$$

where $\hat{\mathbf{n}}_{1}$ is the vector normal on the side of the cube pointing into the body of the cube, $F_{1}(\mathrm{~kg} / \mathrm{s})$ is the flow rate through this side of the cube and $A_{1}\left(\mathrm{~m}^{2}\right)$ is the area of $\Lambda_{1}$. On the opposite side of the cube, $\Lambda_{2}$ the pressure is fixed,

$$
p=p_{2}, \mathbf{x} \in \Gamma_{2}^{C},
$$

where $p_{2}(\mathrm{~Pa})$ is a fixed pressure value. A no-flux condition was applied to all other boundaries. These conditions are summarised in figure 3 A. For the whole lymph node model the condition of afferent lymphatic boundary, $\Gamma_{a}$, was

$$
\hat{\mathbf{n}}_{a} \cdot \rho \mathbf{u}=\frac{F_{a}}{A_{a}}, \mathbf{x} \in \Gamma_{a}^{L},
$$



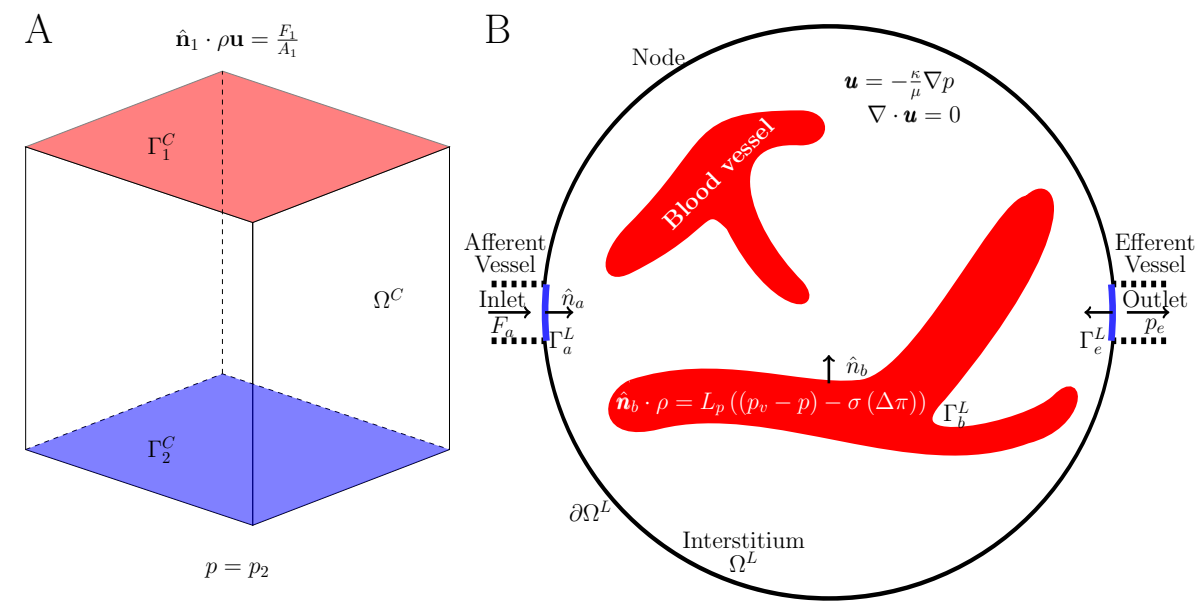

Figure 3: Sketch summarising the cube and whole lymph node models. Dashed black lines show position of afferent and efferent vessels, although these are not modelled. The arrows labelled with $\hat{n}$ show the positive normal vector to the boundaries.

where $\hat{\mathbf{n}}_{a}$ is the vector normal to the afferent lymphatic boundary, pointing into the lymph node, $F_{a}(\mathrm{~kg} / \mathrm{s})$ is the afferent flow rate and $A_{a}\left(\mathrm{~m}^{2}\right)$ is the area of the afferent lymphatic boundary. The pressure is fixed to $p_{e}$ on the efferent lymphatic boundary, $\Gamma_{e}$,

$$
p=p_{e}, \mathbf{x} \in \Gamma_{e}^{L} .
$$

The whole node model is summarised in figure $3 \mathrm{~B}$.

\subsection{Mesh Density Study}

There are some computational choices that needed to be made with regards to how the model is solved numerically, i.e., size of the mesh elements, voxel size of the images and the histogram of the grey scales of the images. To explore the error resulting from these choices, a representative subvolume from the image stack of the whole node was used. This cube was chosen so that it contained one branch of a blood vessel and was $130 \mu \mathrm{m} \times 130 \mu \mathrm{m} \times 130 \mu \mathrm{m}$. Using a subsection of the volume reduced the amount of computational resources required. An inlet flow rate of $F_{1}=5.1 \times 10^{-4} \mathrm{~m} / \mathrm{s}$ was applied to the upper face of the cube and an outlet pressure of $p_{2}=204 \mathrm{~Pa}$ was defined on the lower face. These values 
were evaluated from the two planes $130 \mu \mathrm{m}$ apart through the wild type (WT) node model in Cooper et al. [14.

Two parameters were defined: $\Delta y$, which had a constant value of $2.25 \mu \mathrm{m}$ $\left(\sqrt{3 \times 1.3^{2}} \mu \mathrm{m}\right)$ based on the voxel size of the images; and $\Delta x$ the maximum

185 size of the mesh elements. Initially $\Delta x$ was equal to the minimum element edge length, $2.25 \mu \mathrm{m}$ and then increased by a factor of two six times. This resulted in $\frac{\Delta x}{\Delta y}=32,16,8,4,2$ and 1 .

\subsection{Region Study of Selected Node Structures}

A further study was conducted to investigate the effect of different structures within the node, such as lymphatic channels and varying density within the cortex. Three different regions were used. The regions are highlighted in figure 1. The first region of interest was chosen from an area containing channels, i.e. area with no lymphatic tissue, to be known as 'Channels', figure1B and 11 for Gridrec and Paganin, respectively. The second region was from a densely packed area, known as 'Dense', 11C and 1J for Gridrec and Paganin, respectively. The third region represented the area from the centre of the node, to be called 'Medium', figure 1 $\mathrm{D}$ and $1 \mathrm{~K}$ for Gridrec and Paganin, respectively. The corresponding histograms of the stacks from the Gridrec and Paganin image stacks are also shown in figure 1 .

The results from the mesh density and region studies were used to determine which mesh element size should be used to model the whole node and whether to use the Gridrec or Paganin reconstructed image stack. The results of these studies are presented in sections 3.1 and 3.2

\subsection{Whole Lymph Node Model}

The whole node model was calculated using a constant value for the permeability, for six models with the same external geometry, but with different geometries of blood vessels with different surface areas. This was to investigate the effect of different blood vessel geometries on the pathways of fluid flow through the lymph node. The afferent lymph flow rate was defined as 
$F_{a}=7.6 \times 10^{-7} \mathrm{~kg} / \mathrm{s}$ [1]. The efferent lymph pressure was set to $p_{e}=0 \mathrm{~Pa}$, i.e. everything was measured against atmospheric gauge pressure.

The geometry with 6 branches was used to create a model where the permeability was defined by the images in the Paganin image stack. This was compared to the constant permeability model, with the same blood vessel geometry, to see how the variable permeability affected the flow pathways within the lymph node.

\section{Results}

\subsection{Mesh Density Study}

The results of the mesh density study are shown in figure 4 . The ratio of the
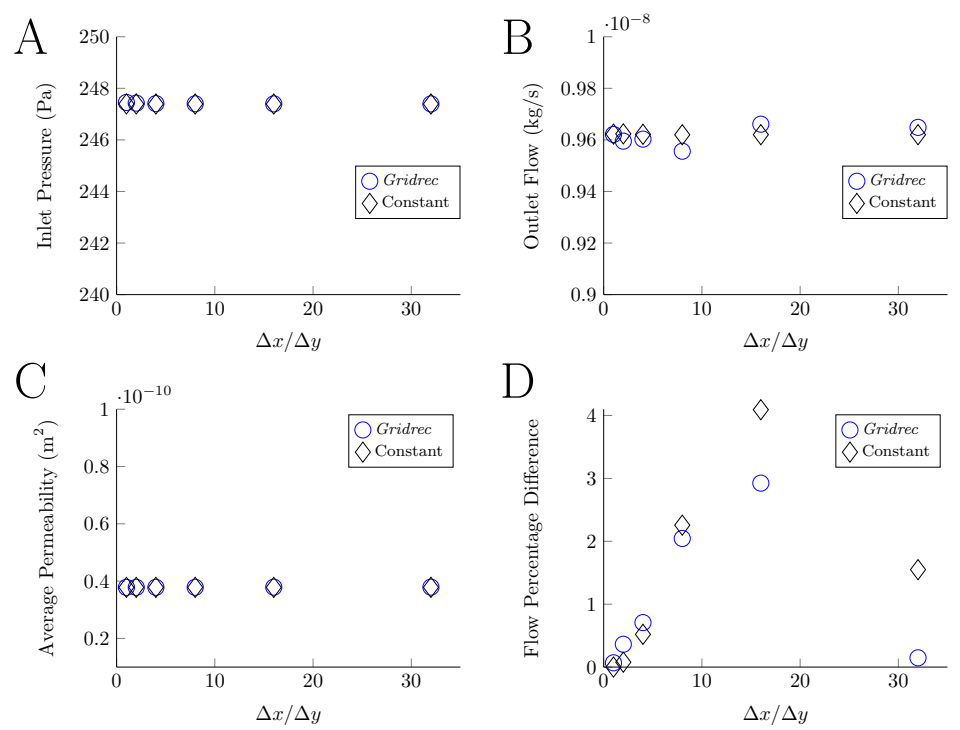

Figure 4: Results of different mesh densities and image stack histograms. A) Inlet; B) Outlet; C) Permeability; D) Flux through central plane.

mesh density to the voxel size, $\Delta x / \Delta y$, did not have a significant effect on the inlet pressure or outlet flow results. However the midplane flow, figure $4 \mathrm{~d}$, is affected. The difference between the mesh with ratio $\Delta x / \Delta y=4$ and the finest mesh have a difference of less than $1 \%$ for the flux through the central plane, 
i.e. well below any experimental error associated with biomedical experiments in this field. Therefore it is suitable to use $\Delta x / \Delta y=4$ for further model simulations presented in this paper.

\subsection{Region Study of Selected Node Structures}

Taking image stacks from different areas of the Gridrec images of the node did not result in much variation for the inlet pressure, outlet pressure or average permeability, figure 5 A, B and C. It did not have the expected effect on the
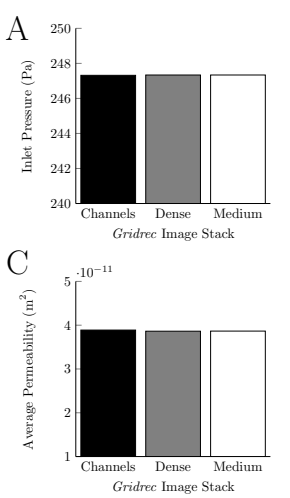
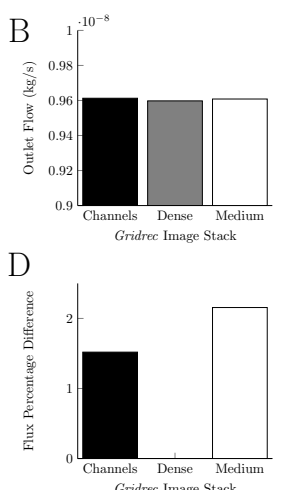
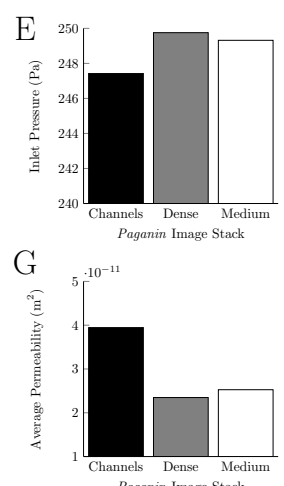

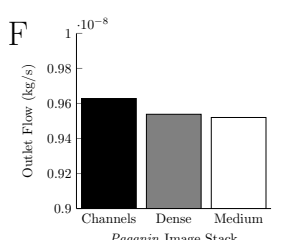

$\mathrm{H}$

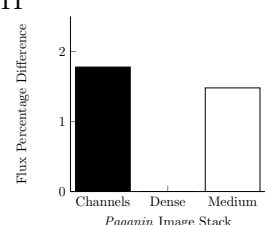

Figure 5: Graphs show how using the image stacks from different regions of the node from the Gridrec and Paganin reconstructions, which were used to define the permeability, affected the flow in a subsection of the node. A and E) Inlet for Gridrec and Paganin reconstructions, respectively; B and F) Outlet for Gridrec and Paganin reconstructions, respectively; C and G) Permeability for Gridrec and Paganin reconstructions, respectively; D and H) Flux through central plane for Gridrec and Paganin reconstructions, respectively. For the Gridrec image stack, the inlet, outlet and average permeability are not affected, but the flux percentage difference is. For the Paganin image stack, the inlet, outlet, average permeability and flux percentage difference are all affected.

230

central cut plane, shown in figure 5D. It was expected that the 'Channels' image stack would allow more flux through than the 'Medium' and 'Dense' stacks, but the results show more flux through the 'Medium' stack. This is probably a result of the very similar grey scale histograms for the image stacks, figures $1 \mathrm{E}$, F and G. It appears that the grey scale of the 'Channels' does not distinguish them as different features. 
Taking stacks from different areas of the Paganin image stack of the node resulted in comparatively more variation for the inlet pressure, outlet pressure, and average permeability, figure $5 \mathrm{E}, \mathrm{F}$ and $\mathrm{G}$. It affected the flow percentage difference through the central cut plane as was expected, see figure $5 \mathrm{H}$, with the most flow though the 'Channels' image stack. The histogram for the Paganin 'Channels' image stack, figure 1l, clearly shows two peaks, one which represents the grey scale of the channels, and the other the grey scale of the surrounding lymphatic tissue.

\subsection{Whole Lymph Node Model}

The whole node model was calculated using a constant value for the permeability for six model set-ups with different blood vessel geometries. Figure 6A, shows how that inlet pressure increased as the surface area of the blood vessels increased. As the surface area of the blood vessel network increases so does the
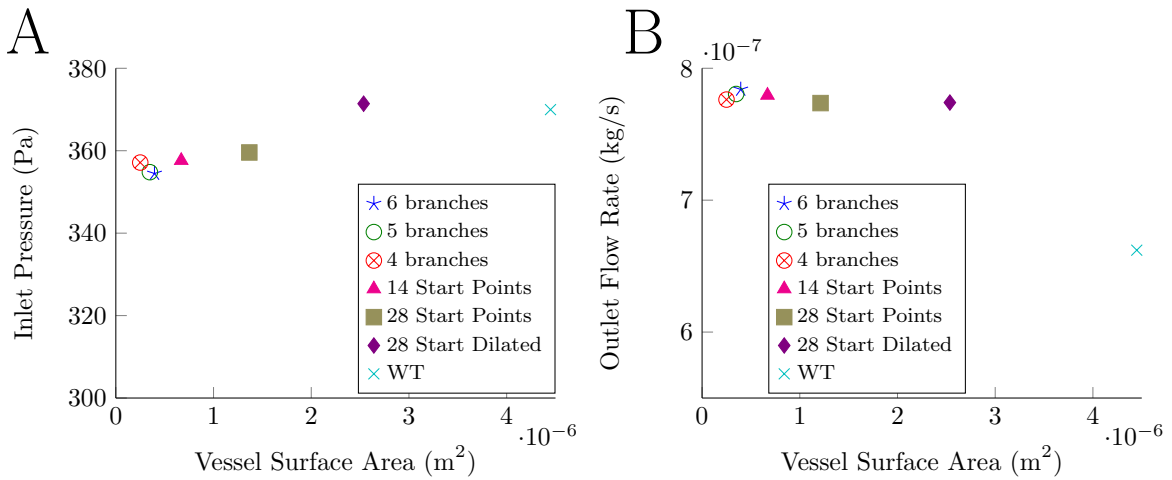

Figure 6: Results showing how the surface area of the blood vessels affects the inlet and outlet flow conditions. A) Inlet pressure; B) Outlet flow rate.

volume. This reduces the cross-sectional area perpendicular to the flow, which effectively increase the overall resistance of the lymph node and therefore increases the afferent pressure required to maintain the outlet flow rate. In figure $6 \mathrm{~B}$, the outlet flow rate is similar for all the blood vessel surface areas. The global value for $L_{p}$ is varied for each model so that all the models have the 

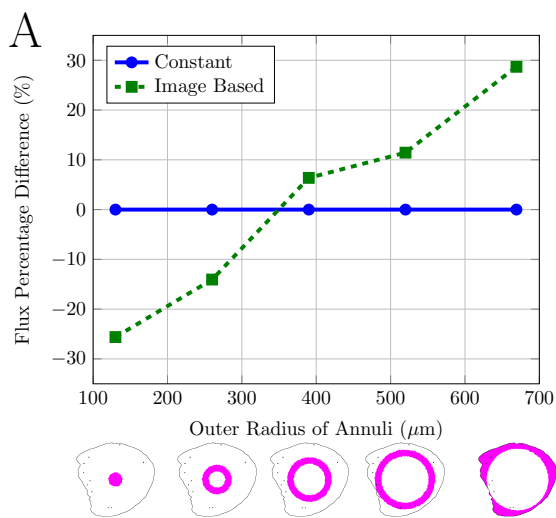

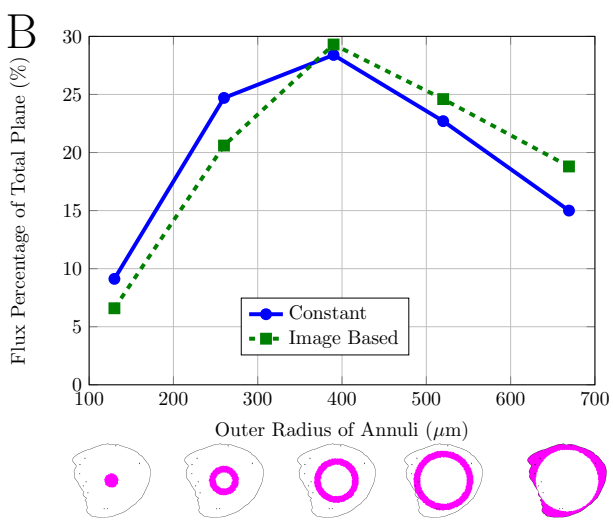

Figure 7: Comparing flux through different annuli (shown in images below graph) through 2D plane equidistant between the inlet and outlet. A) Flux percentage difference through annuli. Values normalised to constant permeability results for comparison, thus all constant values are 0. B) Percentage of the total flux through the plane that passes through each annuli.

flow to take less resistant paths further out. Figure $7 \mathrm{~B}$ shows the percentage of the total flux through the plane that passes through each annuli. This shows that the annuli with the highest percentage of flux is the middle annuli with an outer radius $390 \mu \mathrm{m}$. These results show that incorporating the image based permeability into the model does not cause the majority of the flow to pass around the outer edge of the node, which would be expected if the subcapsular sinus is considered as the path of least resistance. This could indicate the lymph node has shrunk due to the process of fixation and, therefore, the subcapsular sinus has not been fully visualised by the X-ray CT imaging.

Figure 8 shows the comparison between the flow velocity within the node 
between the two models in a plane perpendicular to the inlet and the outlet. The direction and general flow morphology is similar, but that the velocity of the flow is different. The effect of the inlet and outlet can clearly be seen in the flow velocity in the image from the constant permeability model, figure $8 \mathrm{~A}$. The fastest flow takes a direct path between the inlet and the outlet. The image based permeability model similarly shows the majority of the fast flow taking a direct path between the inlet and the outlet but there are some small areas of slower velocities where there is more resistance to the flow due to the variable permeability, figure $8 \mathrm{~B}$.

In planes parallel to that shown in figure 8 , but further from the inlet and outlet, the flow is slower. It is possible to see in the image based permeability model, that near the upper surface, where there are dark areas, the flow is faster than in the same area in the constant permeability model. A similar phenomenon can be observed in the lower quarter of the node. There is a higher velocity in this area in the variable permeability model than in the equivalent area of the constant permeability model. In this area of the node, the presence of medullary sinuses is expected. From descriptions in the literature, this implies that if the subcapsular sinus and medullary sinuses are visualised, they can be incorporated into the model using the methods described above and therefore the model could capture the effect of these structures on the flow pathways.

\section{Discussion}

In this paper a lymph node model has been created using high resolution phase contrast CT images to define the geometry and material properties. A mesh density study has been carried out and it was found that to model the effect of the image based permeability on the flow, it was necessary to have a maximum mesh element edge length no larger than $9 \mu \mathrm{m}$. The minimum element edge length, $2.25 \mu \mathrm{m}$, was based on the diagonal length of a voxel after the original images had been binned. This implies that a mesh element edge 

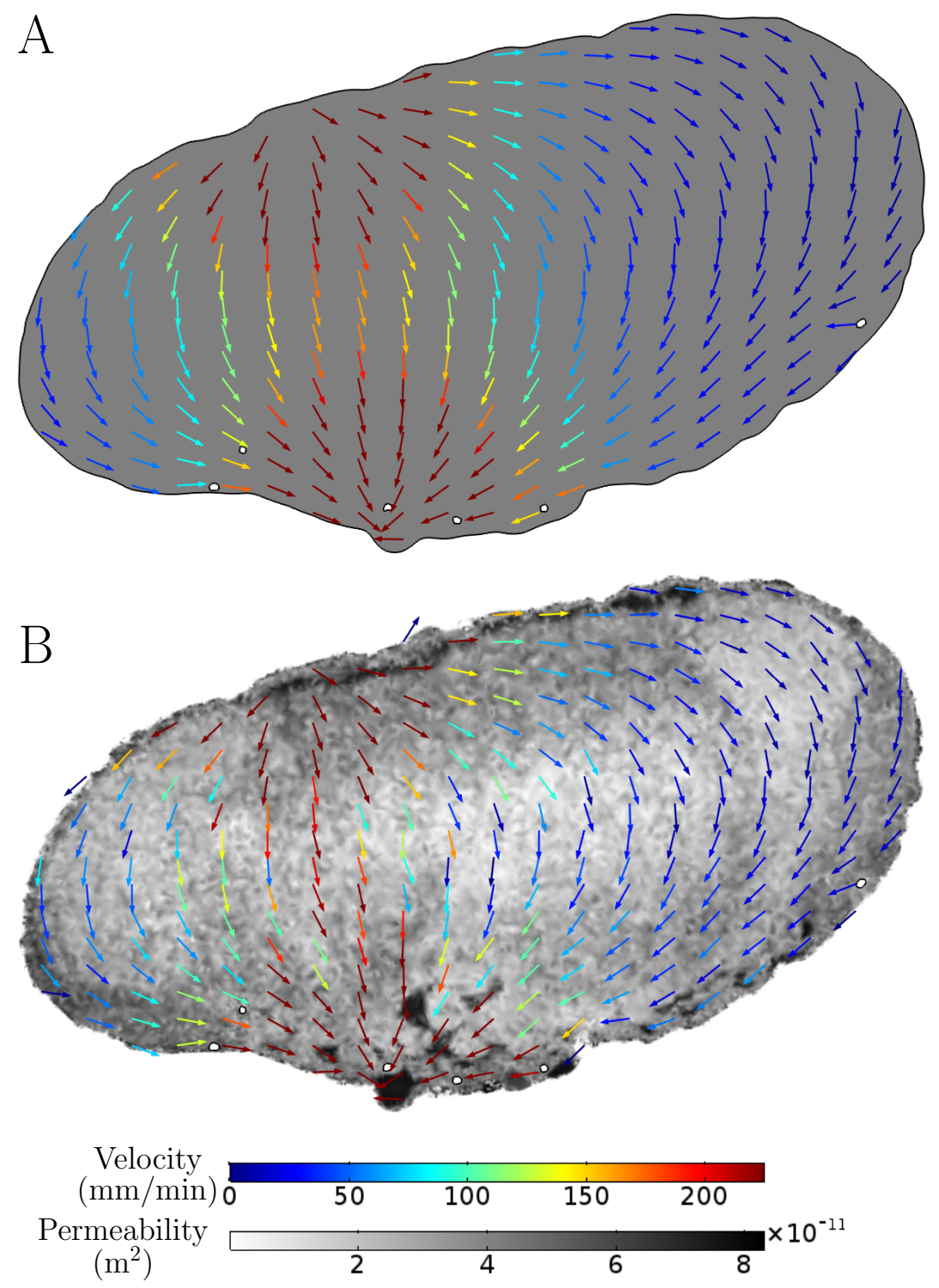

Figure 8: Comparison of constant permeability, A, and image based permeability, B, models showing flow velocity in colour of arrows which indicate the direction of the flow overlaid on the permeability of the plane. 
with less than $1 \%$ error, figure $4 \mathrm{D}$, compared to the mesh with maximum mesh element edge length $2.25 \mu \mathrm{m}$. It has been shown that Paganin phase retrieval prior to tomographic reconstruction produces an image stack that predicts realistic fluid flow profiles. The number and position of the blood vessels does not significantly affect the inlet and outlet conditions of the flow as long as $L_{p} S$ constant. However, it is not clear how the position of blood vessels within the node affects the flow direction and it is important to investigate this further because the blood vessels themselves may act as obstacles to the flow passing through the node. It is known that within the lymph node, blood vessel networks vary in regions with different functions. For example, complex networks of capillaries have been observed just beneath the subcapsular sinus, but blood vessels rarely pass through follicles, instead forming a basket like structure around the outside [4, 2]. The blood vessels may increase resistance to the flow in some regions more than others. The model with Paganin image based permeability resulted in more flow around the outer sections of the node than the constant permeability model. The direction of the flow is similar in the constant and image based permeability models, however the varying permeability affects the velocity of the flow at small scales.

Compared to the results presented in [14], which showed the SPIM image 320 based model resulted in more flow through the centre of the node, the model based on CT images shows that the structure of the node causes more flux through the outer section of the node. In the CT images, a lot more of the internal node structure is visible compared to the SPIM images, where only the stained areas had been visualised. Thus, the more detailed structure from the CT scans is likely to lead to more detailed results. However, the X-ray $\mathrm{CT}$ images are not able to visualise all the structures that would be expected within the lymph node, such as blood vessels, due to shrinkage of the sample during fixation and lack of contrast, which makes segmentation challenging. It is also not possible to identify relationships between structure and function in 330 the lymph node using the CT images alone. Both the results presented here and the results in Cooper et al. 14 are based on a linear relation between the 
grey scale of the images and the permeability. This was chosen as the simplest way to relate the two values. It would be possible to relate the grey scale to the permeability with a non-linear relation. However, the model can only be

voxel size of the SPIM or confocal microscopy imaging need to be high enough to capture the smallest blood vessels, capillaries, which are approximately $5-10 \mu \mathrm{m}$ 
in diameter. Therefore, to visualise the capillaries it will be necessary to have a minimum mesh element size that can capture this geometry and a maximum element size equivalent to the voxel size of the CT images. This information will be useful for planning further imaging and modelling work. Combining SPIM with X-ray CT would allow a step change in our ability to model real biological geometries while also being able to utilise the information we gain about the material properties from imaging. In the future, it would also be useful to perform histology on the same lymph node to compare if the structures that can be observed in the CT images can be linked to function or cell types. This would also enable a region analysis in 3D to compare the difference in volume of the various structures in the lymph node. Within lymph nodes there are many different functional scales. Lymphocytes measure approximately $10 \mu \mathrm{m}$ in di375 ameter [25] and make up the majority of the lymphatic tissue. Veins in murine lymph nodes can have diameters up to $150 \mu \mathrm{m}$ [4]. The size of paracortex and B cell follicles can vary depending on the quantity of lymphocytes with in the nodes. The paracortex can make up $50 \%$ of lymph node tissue and therefore have diameters of $0.5-1 \mathrm{~mm}$ in murine lymph nodes. Although the resolution of the images used here, $1.3 \mu \mathrm{m}^{3}$, should mean that it is possible to distinguish these structures, without correlating this with histology or SPIM it is not possible to validate that the structures visualised due to density variations are indeed the functional structures previously identified by biologists.

This phenomenon could be further investigated if images of an unfixed, hydrated lymph node could be obtained to overcome the issues of shrinkage during fixation. Practically, this would require imaging at a synchrotron lightsource and with a temperature-controlled environment. Recently, in vivo imaging has been carried out [26]. If the subcapsular sinus and lymphatic channels could be visualised and segmented, a useful alternative to Darcys law would be the Brinkman equation. However, this would introduce an extra parameter, which would be challenging to validate. If the subcapsular sinus and lymphatic channels could be visualised and segmented, applying the Brinkman equation may result in an increase in the velocity of the flow around the outer edges of the 
node where, in the present model, the slowest flow is observed.

ton, EPSRC 1230417. We acknowledge the Paul Scherrer Institut, Villigen, Switzerland for provision of synchrotron radiation beamtime at the TOMCAT 
beamline of the SLS and would like to thank Pablo Villanueva and Alessandra Patera for their assistance. The authors acknowledge the use of the IRIDIS High Performance Computing Facility and the $\mu$-VIS Centre for Computed Tomography, at the University of Southampton in the completion of this work.

\section{References}

[1] Y. Ohtani, B. jun Wang, R. Poonkhum, O. Ohtani, Pathways for movement of fluid and cells from hepatic sinusoids to the portal lymphatic vessels and subcapsular region in rat livers, Archives of Histology and Cytology 66 (2003) 239-252.

[2] M. C. Kowala, G. I. Schoefl, The popliteal lymph node of the mouse: internal architecture, vascular distribution and lymphatic supply, J Anat 148 (1986) 25-46.

[3] C. L. Willard-Mack, Normal structure, function, and histology of lymph nodes, Toxicol Pathol 34 (2006) 409-24.

[4] A. O. Anderson, N. D. Anderson, Studies on the structure and permeability of the microvasculature in normal rat lymph nodes, Am J Pathol 80 (1975) 387-418.

[5] O. Ohtani, Y. Ohtani, Structure and function of rat lymph nodes, Archives of Histology and Cytology 71 (2008) 69-76.

[6] A. A. Tomei, S. Siegert, M. R. Britschgi, S. A. Luther, M. A. Swartz, Fluid flow regulates stromal cell organization and ccl21 expression in a tissueengineered lymph node microenvironment, The Journal of Immunology 183(7) (2009) 4273-4283.

[7] T. Nagai, F. Ikomi, S. Suzuki, T. Ohhashi, In situ lymph dynamic characterization through lymph nodes in rabbit hind leg: Special reference to nodal inflammation., The Journal of Physiological Sciences 58 (2008) 123132. 
[8] I. L. Grigorova, M. Panteleev, J. G. Cyster, Lymph node cortical sinus organization and relationship to lymphocyte egress dynamics and antigen exposure, Proceedings of the National Academy of Sciences 107 (2010) 20447-20452.

[9] T. H. Adair, D. S. Moffatt, A. W. Paulsen, A. C. Guyton, Quatitiation of changes in lymph protein concentration during lymph node transit, American Journal of Physiology: Heart and Circulation Physiology 243 (1982) H351-H359.

[10] T. H. Adair, A. C. Guyton, Modification of lymph by lymph nodes. ii. effect of increased lymph node venous blood pressure, American Journal of Physiology: Heart and Circulation Physiology 245 (1983) H616-H622.

[11] T. H. Adair, A. C. Guyton, Modification of lymph by lymph nodes iii. effect of increased lymph hydrostatic pressure, American Journal of Physiology: Heart and Circulation Physiology 249 (1985) H777-H782.

[12] M. A. Swartz, M. Skobe, Lymphatic function, lymphangiogenesis and cancer metastasis, Microscopy Research and Technique 55 (2001) 92-99.

[13] J. Mayer, J. Swoger, A. J. Ozga, J. V. Stein, J. Sharpe, Quantitative measurements in 3-dimensional datasets of mouse lymph nodes resolve organwide functional dependencies, Comput Math Methods Med 2012 (2012) 128431.

[14] L. J. Cooper, J. P. Heppell, G. F. Clough, B. Ganapathisubramani, T. Roose, An image based model of fluid flow through lymph nodes, Bulletin of Mathematical Biology 78(1) (2016) 52-71.

[15] B. Zeller-Plumhoff, T. Roose, G. F. Clough, P. Schneider, Image-based modelling of skeletal muscle oxygenation, Journal of The Royal Society Interface 14 (2017).

[16] B. Zeller-Plumhoff, K. R. Daly, G. F. Clough, P. Schneider, T. Roose, Investigation of microvascular morphological measures for skeletal muscle 
tissue oxygenation by image-based modelling in three dimensions, Journal of The Royal Society Interface 14 (2017).

[17] F. Marone, M. Stampanoni, Regridding reconstruction algorithm for realtime tomographic imaging, J Synchrotron Radiat 19 (2012) 1029-37.

[18] B. A. Dowd, G. H. Campbell, R. B. Marr, V. Nagarkar, S. Tipnis, L. Axe, D. P. Siddons, Developments in synchrotron x-ray computed microtomography at the national synchrotron light source., Developments in X-Ray Tomography II 3772 (1999) 224-236.

[19] D. Paganin, S. C. Mayo, T. E. Gureyev, P. R. Miller, S. W. Wilkins, Simultaneous phase and amplitude extraction from a single defocused image of a homogeneous object, Journal of Microscopy 206 (2002) 33-40.

[20] J. Schindelin, I. Arganda-Carreras, E. Frise, V. Kaynig, M. Longair, T. Pietzsch, S. Preibisch, C. Rueden, S. Saalfeld, B. Schmid, J.-Y. Tinevez, D. J. White, V. Hartenstein, K. Eliceiri, P. Tomancak, A. Cardona, Fiji: an open-source platform for biological-image analysis, Nature Methods 9 (2012) 676-682.

[21] R. Burton-Opitz, R. Nemser, The viscosity of lymph, American Journal of Physiology - Legacy Content 45 (1917) 25-29.

[22] J. R. Levick, An introduction to cardiovascular physiology, 5th ed. ed., London : Hodder Arnold, 2009.

[23] M. Stohrer, Y. Boucher, M. Stangassinger, R. K. Jain, Oncotic pressure in solid tumors is elevated, Cancer Research 60 (2000) 4251-4255.

[24] M. Jafarnejad, M. Woodruff, D. Zawieja, M. Carroll, J. E. Moore, Jr., Modeling lymph flow and fluid exchange with blood vessels in lymph nodes, Lymphatic Research and Biology 13 (2015) 234-245.

[25] A. S. Perelson, F. W. Wiegel, Scaling aspects of lymphocyte trafficking, Journal of Theoretical Biology 7 (2009) 9-16. 
[26] G. Lovric, R. Mokso, F. Arcadu, I. V. Oikonomidis, J. C. Schittny, M. RothKleiner, M. Stampanoni, Tomographic in vivo microscopy for the study of lung physiology at the alveolar level, Scientific Reports 7 (2017).

[27] P. Prusinkiewicz, J. Hanan, Lindenmayer systems, fractals, and plants, Berlin : Springer, 1989.

[28] M. Zamir, Arterial branching within the confines of fractal l-system formalism, Journal of General Physiology 118 (2001) 267-275.

[29] M. A. Galarreta-Valverde, M. M. G. Macedo, C. M. an dMarcel P. Jackowski, Three-dimensional synthetic blood vessel generation using stochastic l-systems, Proc. SPIE 8669, Medical Imaging 2013: Image Processing 8669 (2013) 86691I-1-86691I-6.

\section{Appendix A. Fractal L-system}

A fractal L-system 27] was used to create the 3D blood vessel architecture within the node. An L-system is a language where strings of symbols are interpreted sequentially to create fractal geometries. The fractal L-system method

520 summarised here is from the methods created by Zamir [28] and GalarretaValverde et al. [29].

For a fractal L-system implementation, rules are created to define the branching structure. Each character represents an instruction. Two rules are used to describe the blood vessel structure, one to describe the horizontal splitting and one to describe the vertical splitting. The first is

$$
X \mapsto F[-Y][+Y]
$$

where the characters represent the following instructions: $X$ and $Y$ act as place holders, i.e. the respective rule can be substituted in to extend the sequence, $F$ means move forward a defined distance, [ means save the current position, - and + mean rotate by $\theta$ degrees clockwise or anticlockwise, respectively, in 
the current plane, and ] means return to the previous position. Effectively, this creates a branch that bifurcates horizontally. The second rule is

$$
Y \mapsto F[/ X][* X]
$$

where / and $*$ mean rotate by $\theta$ degrees clockwise or anticlockwise, respectively, in the plane perpendicular to the current plane. This creates a branch that bifurcates vertically. $\theta$ is defined as

$$
\theta=\arccos \left(\frac{\left(\left(1+\alpha^{3}\right)^{4 / 3}+1-\alpha^{4}\right)}{\left(2\left(1+\alpha^{3}\right)^{2 / 3}\right)}\right)=0.654
$$

where $\alpha=1$. This causes the branching structure created to be symmetrical. The points of the segmented vessels ends are used as the start points for growing the blood vessels trees. Another point was found approximately $10 \mu \mathrm{m}$ back along the vessel length and the unit vector corresponding to the vector between these two points was defined as the heading vector. The first plane is parallel to a slice from the image stack.

The rules are substituted into one another repeatedly to create the required number of branch bifurcations. An example of the rules used for different branch bifurcations are shown in table A.1

\begin{tabular}{|c|l|}
\hline Bifurcations & Rule \\
\hline 1 & $F[-Y][+Y]$ \\
2 & $F[-F[/ X][* X]][+F[/ X][* X]]$ \\
3 & $F[-F[/ F[-Y][+Y]][* F[-Y][+Y]]][+F[/ F[-Y][+Y]][* F[-Y][+Y]]]$ \\
\hline
\end{tabular}

Table A.1: Rules used to create blood vessel trees for different numbers of bifurcations. Each character is translated to create the geometry: $X$ and $Y$, place holders, $F$, move forward one step, [, save the current position, - and + , rotate by angle $\theta$ clockwise or anticlockwise, respectively, in the current plane, and ], return to the previous position, / and $*$, rotate by $\theta$ degrees clockwise or anticlockwise, respectively, in the plane perpendicular to the current plane.

Before a new branch is created, the algorithm checks whether the new end point is inside the node by checking if the new point has the value to 1 (white) 
in the mask of the image stack that represents the geometry of the node, the segmentation of this is described in 2.3 . The algorithm also checks to see if the new branch intersects with any other branches of the same tree by solving the simultaneous equations,

$$
\begin{aligned}
& \mathbf{x}_{\text {start }}+a \mathbf{x}_{\text {end }}=\mathbf{p} \\
& \mathbf{x}_{\text {start }}^{i}+b \mathbf{x}_{\text {end }}^{i}=\mathbf{p}
\end{aligned}
$$

where $\mathbf{x}_{\text {start }}$ and $\mathbf{x}_{\text {end }}$ are the start and end points of the new branch, respectively, $a$ is an unknown parameter, and $p$ is the point of intersection, $\mathbf{x}_{\text {start }}$ and $\mathbf{x}_{\text {end }}$ are the start and end points of branch $i$, and $b$ is another unknown parameter. If the new branch and branch $i$ intersect, then $\mathbf{p}$ is the point of intersection and $a$ and $b$ are the parameters that calculate that point. If the branches do not intersect then $\mathbf{p}, a$ and $b$ are returned as empty values.

If the branches are found to intersect it is further checked to see if this intersection occurs at the end of branch $i$. If it does, then the new branch is saved and the next iteration begins. If the new branch intersects with the beginning of branch $i$, another check is made to ensure that the new branch will not be the third branch initiated at that point, since the tree should only develop bifurcations. If all of these checks are passed, the final check is to find out if the intersection occurs within the length of the branch. This is check be calculating,

$$
c_{1}=\left(\mathbf{x}_{\text {start }}^{i}-\mathbf{x}_{\text {end }}^{i}\right) \cdot\left(\mathbf{p}-\mathbf{x}_{\text {start }}^{i}\right)
$$

If $c_{1}$ is less than zero, the intersection occurs before the start point and the new branch can be saved. However, if it is greater than zero, the dot product between the start and end points of branch $i$ and between the intersection point and the end of branch $i$ must also be found, i.e.,

$$
c_{2}=\left(\mathbf{x}_{\text {start }}^{i}-\mathbf{x}_{\text {end }}^{i}\right) \cdot\left(\mathbf{p}-\mathbf{x}_{\text {end }}^{i}\right)
$$

If $c_{1}$ is greater than $c_{2}$, the intersection occurs after the end point and the new branch can be saved. However, if not the intersection occurs within the length 
of the branch. If the new branch fails this, or any of the previous checks the 540 new branch is discarded and the next new branch is initiated. 